\title{
EUM: Antihipertensivos en la Seguridad Social y Análisis Comparativo entre Centros de Atención Médica Ambulatoria
}

\author{
Desirée Sáenz-Campos, ${ }^{1}$ Zahira Tinoco-Mora, ${ }^{1}$ Laura Rojas-Mora ${ }^{1}$
}

Justificación: La Hipertensión Arterial es una enfermedad crónica y asintómatica, ocupa la primera causa de consulta médica ambulatoria, contribuye directamente con la primera causa de mortalidad en el país, y casi siempre es fácil de tratar con una intervención integral que incluye la terapia farmacológica.

Objetivo: Identificar los fármacos con mayor consumo institucional, valorar si el perfil resultante es compatible con los principios del uso racional de antihipertensivos y comparar la utilización de estos agentes entre clínicas similares de atención médica ambulatoria, durante un periodo anual.

Métodos: Estudio observacional de tipo farmacoepidemiológico, se obtuvo el registro del consumo institucional de cada antihipertensivo y reporte local de consumo mensual de seis centros de atención médica ambulatoria, durante 1999. Se calculó dosis diaria definida (DDD) y estimación de pacientes tratados y se hizo un análisis estadístico descriptivo.

Resultados: Los fármacos más prescritos fueron atenolol (25\%), enalapril (21\%) e hidroclorotiacida (19\%); según DDD, el consumo global de atenolol fue $39 \%$, enalapril $25 \%$ e hidroclorotiacida $23 \%$, con lo que se benefició el $87 \%$ de los pacientes tratados. Con los antihipertensivos se dio tratamiento a unos 185,639 pacientes. Al nivel local, los perfiles de consumo tienden a simular la distribución institucional al predominar el uso de atenolol pero se alterna el diurético con enalapril; se registraron variaciones en el consumo mensual durante el periodo y en la cobertura de pacientes hipertensos de la zona.

Conclusiones: Todos los fármacos antihipertensivos disponibles son utilizados aunque su consumo muestra un perfil heterogéneo. Los fármacos más prescritos al nivel local replican el perfil de consumo institucional, pero hay notables variaciones locales. Los hábitos de prescripción se ajustan al uso racional de los antihipertensivos en el ámbito ambulatorio. Las estimaciones derivadas del consumo sugieren una gran proporción de pacientes sin control, o con tratamiento fuera de la Seguridad Social.

Descriptores: Estudios de utilización de medicamentos, hipertensión arterial, atenolol, enalapril, hidroclorotiacida.

Recibido: 13 de octubre de 2000

Acepatado para publicación: 13 de febrero de 2001

\begin{abstract}
Abreviaturas: CCSS, Caja Costarricense de Seguro Social; EUM, Estudios de Utilización de Medicamentos; HTA, Hipertensión Arterial; SS, Seguridad Social; UCR, Universidad de Costa Rica.

1 Grupo de investigación para Estudios de Utilización de Medicamentos, proyecto UCR-CCSS: VI-422-A0-138 (Escuela de Medicina, UCR; Dpto. Farmacoterapia, CCSS).
\end{abstract}

Correspondencia: Desirée Sáenz Campos. Dpto. Farmacoterapia, Oficinas centrales CCSS, piso 12; San José, Costa Rica. Teléfono (506) 222-1878, 295-2267; fax (506)257-7004.

\section{Introducción}

La Hipertensión Arterial (HTA) es una enfermedad frecuente, crónica, asintomática, fácil de detectar, casi siempre fácil de tratar y que lleva a complicaciones importantes cuando no se controla. ${ }^{1}$ Conforma un problema de salud tan relevante que, desde hace años, ocupa la primera causa de consulta médica ambulatoria de adultos en la Seguridad Social (SS) y contribuye directamente a la primera causa de mortalidad en el país. ${ }^{2}$

Como sucede con casi toda condición mórbida, el control y manejo terapéutico de la HTA requiere de un abordaje integral que va más allá del sólo descenso de los niveles de la 
presión arterial. La aplicación de medidas no farmacológicas, tales como disminuir el peso si éste es excesivo, incrementar la actividad física aeróbica, limitar la ingesta etílica a un máximo de $30 \mathrm{~mL}$ de etanol/día en varones y $15 \mathrm{~mL} /$ día en mujeres, suspender tabaquismo, disminuir el consumo de sal a $6 \mathrm{~g}$ /día, mantener una adecuada ingesta de potasio, calcio y magnesio; reducir la ingestión alimentaria de grasas saturadas y colesterol, procurar la relajación y el descanso óptimo son tan importantes como la intervención farmacológica, ${ }^{3,4}$ para la cual, si es requerida, se emplean los antihipertensivos. $\mathrm{Al}$ efecto, se dispone de múltiples agentes farmacológicos que se diferencian por composición química, mecanismo de acción y posología, además de otras características muy relevantes como tolerabilidad, eficacia demostrada a largo plazo, contraindicaciones y costo.

Para el manejo farmacológico de la HTA, la SS ofrece una variedad limitada de medicamentos. Tal como especifica su Lista Oficial de Medicamentos, para uso por vía oral se cuenta con 7 medicamentos distintos para la HTA como primera indicación, ${ }^{6}$ presentados como "Grupo 08 Antihipertensores" y uno más en el "Grupo 10 Vasodilatadores". ${ }^{5}$ El médico tiene la responsabilidad de la selección individual entre las alternativas disponibles para la prescripción óptima.

Dada la relevancia que tienen los medicamentos para el tratamiento ambulatorio de la HTA, los objetivos del presente estudio son identificar los fármacos con mayor consumo a nivel institucional, valorar si el perfil resultante es compatible con los principios del uso racional de antihipertensivos y comparar la utilización de estos agentes entre clínicas similares de atención médica ambulatoria, durante un periodo anual.

\section{Materiales y Métodos}

El estudio fue observacional, descriptivo, transversal, de tipo farmacoepidemiológico, en un entorno de práctica médica habitual, multicéntrico y comparativo entre centros. El consumo institucional de cada uno de los antihipertensivos se obtuvo del Informe de Consumo y Costos 1999 de la SS; ${ }^{6}$ los de cada centro fueron suplidos individualmente por los Servicios de Farmacia (Informe Mensual del Consumo de Antihipertensivos, periodo 1999) de los siguientes sitios: Dr. Carlos Durán, Dr. Solón Núñez, Integrada de Tibás, Palmares, Area Flores-Belén y San Rafael de Heredia.

Los datos se digitaron en una matriz de datos; para calcular la dosis diaria definida (DDD) se aplicó la fórmula[(mg/ uni- dad*unidades consumidas)/DDD (mg)*1000habitantes/ $(3.543,389$ cobertura $* 365$ días)] para la estandarización de resultados, ${ }^{7}$ ajustados según población cubierta $(98 \%)$ para estimar el número de pacientes tratados; la población general se obtuvo del Sistema Integrado de Información del Registro Civil y la cubierta por cada clínica con Registros Biomédicos de cada lugar (vía telefónica). Para la comparación de los consumos de antihipertensivos entre centros de atención médica, se procedió al análisis bajo una aproximación descriptiva.

\section{Resultados}

Durante el periodo de 1999 y en el ámbito institucional, en conjunto los pacientes recibieron más de 80 millones de tabletas de antihipertensivos. En orden decreciente, los fármacos más prescritos fueron atenolol $(25.0 \%)$, enalapril $(21.4 \%)$, hidroclorotiacida (19.1\%) y nifedipino $(18.2 \%)$.

\section{Cuadro 1 \\ Consumo anual de antihipertensivos en la SS, consumo diario absoluto, consumo en DDD y estimación general de pacientes tratados (periodo 1999)}

\begin{tabular}{|c|c|c|c|c|c|c|}
\hline Fármaco & $\begin{array}{c}\text { Consumo anual } \\
\text { (tabletas) }\end{array}$ & $\begin{array}{c}\text { Consumo diario } \\
\text { (tabletas) }\end{array}$ & $\begin{array}{l}\text { DDD } \\
\text { (mg) }\end{array}$ & $\begin{array}{c}\mathrm{DDD} / 1000 \\
\text { habitantes/día }\end{array}$ & $\begin{array}{c}\text { Pacientes/día* } \\
\text { (estimación) }\end{array}$ & $\%$ \\
\hline $\begin{array}{l}\text { 08-0210 Atenolol, } \\
\text { tabletas de } 100 \mathrm{mg}\end{array}$ & $20.002,150$ & 54,801 & 75 & 20.62 & 73,067 & 39.4 \\
\hline $\begin{array}{l}\text { 10-1220 Nifedipino, } \\
\text { cápsulas de } 10 \text { mg }\end{array}$ & $14.577,150$ & 39,938 & 30 & 3.76 & 13,313 & 7.2 \\
\hline $\begin{array}{l}\text { 08-0267 Enalaprila, } \\
\text { tabletas de } 20 \mathrm{mg}\end{array}$ & $17.145,900$ & 46,976 & 20 & 13.26 & 46,975 & 25.3 \\
\hline $\begin{array}{l}\text { 08-0840 Hidralazina, } \\
\text { tabletas de } 50 \mathrm{mg}\end{array}$ & 737,380 & 2,021 & 100 & 0.29 & 1,010 & 0.5 \\
\hline $\begin{array}{l}\text { 08-0850 Hidroclorotia- } \\
\text { cida, tabletas de } 25 \mathrm{mg}\end{array}$ & $15.290,530$ & 41892 & 25 & 11.82 & 41,892 & 22.6 \\
\hline $\begin{array}{l}\text { 08-1030 Metildopa, } \\
\text { tabletas de } 250 \mathrm{mg}\end{array}$ & $4.772,860$ & 13,077 & 1,000 & 0.92 & 3,269 & 1.8 \\
\hline $\begin{array}{l}\text { 08-1040 Metildopa, } \\
\text { tabletas de } 500 \mathrm{mg}\end{array}$ & $1.332,830$ & 3,652 & 1,000 & 0.52 & 1,826 & 1.0 \\
\hline $\begin{array}{l}\text { 08-1520 Propranolol, } \\
\text { tabletas de } 40 \mathrm{mg}\end{array}$ & $6.258,880$ & 17,148 & 160 & 1.21 & 4,287 & 2.2 \\
\hline Total & $80.117,680$ & 219,501 & - & 52.39 & 185,639 & 100 \\
\hline
\end{tabular}


Con las estimaciones del manejo de la HTA en el contexto normatizado de la metodología DDD (Cuadro 1), destacó el consumo global de atenolol con un $39.4 \%$, seguido por enalapril con $25.3 \%$ e hidroclorotiazida con un $22.6 \%$; en tanto que el nifedipino ocupó un lugar marginal (7.2\%). Además, como consecuencia de una prescripción fútil, el consumo de metildopa en sus dos presentaciones fue de tan solo $2.7 \%$, seguido por hidralacina $(0.55 \%)$.

El consumo anual de antihipertensivos alcanza tal magnitud que permitió estimar una cobertura farmacológica de 185,639 personas, con un esquema básico de monoterapia. En consecuencia, con los tres fármacos más prescritos (atenolol, enalapril e hidroclorotiacida) se estimó el tratamiento farmacológico para unas 161,934 personas, lo cual representa un $87.3 \%$ de esos pacientes tratados por vía SS.

Además, al acumular el consumo de los beta-bloqueadores como grupo (atenolol+propranolol= 21.83 DDD/1000habitantes/día), se estimó un suplimiento farmacológico para el $41.7 \%$ de los pacientes; aunque el propranolol se dispuso solo para un $3.5 \%$ de los pacientes. Asimismo, al agrupar los beta-bloqueadores con hidroclorotiacida, se amplió a un $64.3 \%$ de los pacientes.

Por otra parte, se encontró que tanto el consumo local de antihipertensivos en los diferentes centros de atención médica como la proporción poblacional que recibió el tratamiento son cuantitativamente diferentes (Cuadro 2), a pesar de contar con características similares en cuanto a la atención médica ambulatoria. Primero, en las clínicas mayores, descritas tradicionalmente como de tipo 4, se encontró que el consumo de antihipertensivos en la clínica de Tibás duplicó el de la clínica Carlos Durán con 60.62 DDDs frente a 29.28 DDDs, en tanto que se pudo estimar en 3505 y 4527 las personas tratadas, lo cual implica una cobertura en torno al $6.7 \%$ y $2.9 \%$ de la población local, respectivamente. En cuanto a estos resultados, la clínica Solón Nuñez ocupó un lugar intermedio.

Segundo, en torno a las Areas de Salud (originalmente clínicas tipo 3), Flores-Belén con unos 3019 hipertensos tratados (un $9.0 \%$ de la población) y 90.11 DDDs, duplicó a San Rafael en cuanto al consumo global de antihipertensivos (42.44 DDDs) y al número de pacientes tratados (1699 personas, $4.2 \%$ de la población local); y, casi triplicó a Palmares, en donde se brinda tratamiento al $3.7 \%$ de la población y se tuvo un consumo anual de 36.51 DDDs.

Al considerar el consumo particular por fármaco en los diferentes centros de atención médica, los perfiles de utilización son disímiles entre las distintas localidades (Figura 1), aunque la diferencia resultó menos acusada respecto al consumo global registrado para la institución. En este contexto, el atenolol ocupó un primer lugar de forma sistemática,

\begin{tabular}{|c|c|c|c|c|c|c|}
\hline \multicolumn{7}{|c|}{$\begin{array}{c}\text { Cuadro } 2 \\
\text { Consumo anual de antihipertensivos en } 6 \text { clínicas de la CCSS (absoluto y según DDD) } \\
\text { y estimación de pacientes tratados (periodo 1999) }\end{array}$} \\
\hline Medicamento & C. Durán & Int-Tibás & S.Núñez & Palmares & Flores-Belén & S.Rafael-Hdia \\
\hline Atenolol 100mg & 387,098 & 367,167 & 337,730 & 139,484 & 296,351 & 164,847 \\
\hline Hidralazina 50mg & 11,160 & 11,412 & 3,655 & 5,930 & 13,710 & 5,345 \\
\hline Hidroclorotiazida 25mg & 294,130 & 244,560 & 167,852 & 72,671 & 149,937 & 208,358 \\
\hline Metildopa 250mg & 33,070 & 33,660 & 109,161 & 60,631 & 44,666 & 42,533 \\
\hline Metildopa 500mg & 11,515 & 16,908 & 23,205 & 13,570 & 15,727 & 27,770 \\
\hline Propranolol 40mg & 69,896 & 88,680 & 56,610 & 21,896 & 75,881 & 34,371 \\
\hline Población cubierta 1999 & 154,599 & 57,814 & 60,000 & 30,919 & 33,500 & 40,029 \\
\hline Atenolol 100mg & 9.15 & 23.20 & 20.56 & 16.48 & 32.32 & 15.04 \\
\hline Enalapril 20mg & 12.07 & 19.46 & 13.53 & 7.44 & 31.41 & 7.98 \\
\hline Hidralazina 50mg & 0.10 & 0.27 & 0.08 & 0.26 & 0.56 & 0.18 \\
\hline Hidroclorotiazida 25mg & 5.21 & 11.59 & 7.66 & 6.44 & 12.26 & 14.26 \\
\hline Metildopa 250+500mg & 0.25 & 0.80 & 1.78 & 1.94 & 1.55 & 1.69 \\
\hline Propranolol 40mg & 0.31 & 1.05 & 0.65 & 0.49 & 1.55 & 0.59 \\
\hline Nifedipina 10mg & 2.19 & 4.25 & 2.87 & 3.46 & 10.45 & 2.70 \\
\hline Total DDD & 29.28 & 60.62 & 47.14 & 36.51 & 90.11 & 42.44 \\
\hline Pacientes tratados & 4527 & 3505 & 2829 & 1129 & 3019 & 1699 \\
\hline
\end{tabular}




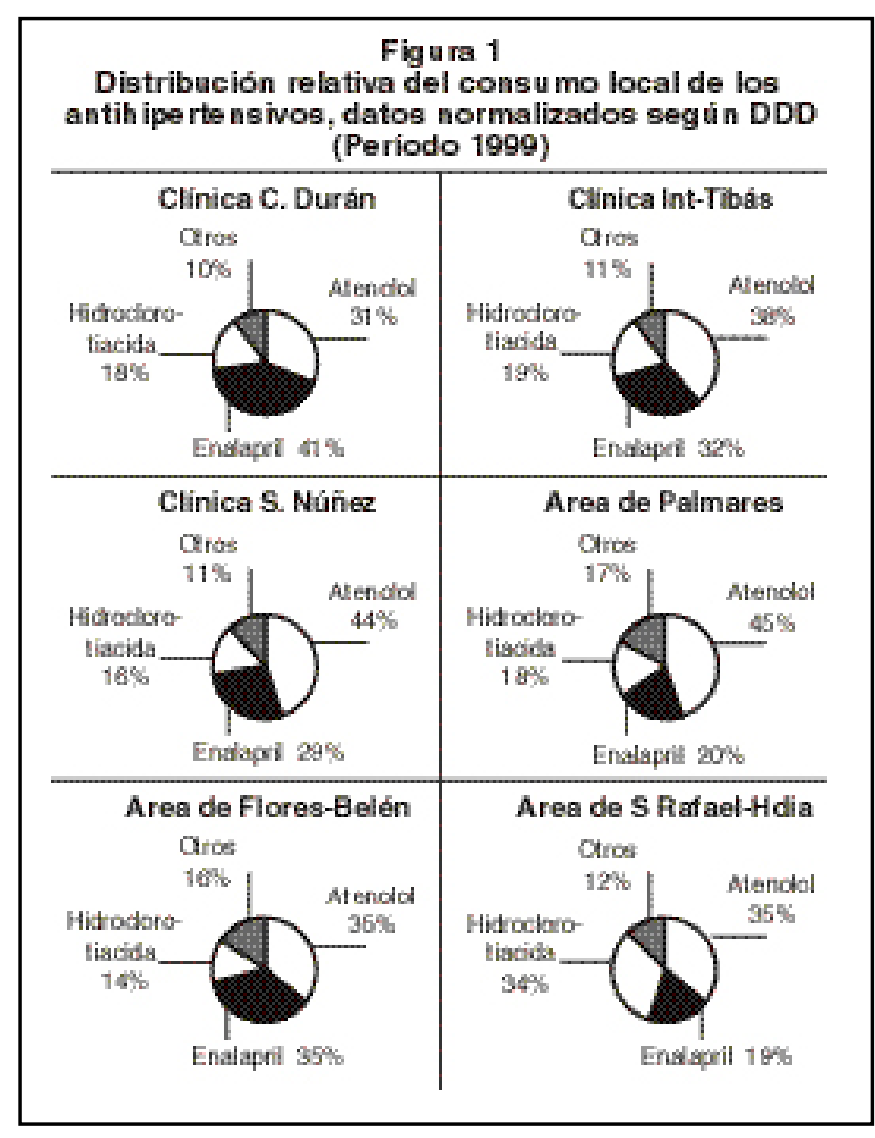

pero tuvo una excepción notable: la Clínica Carlos Durán, donde se identificó al enalapril como el antihipertensivo más prescrito, tanto que supera con un $+10 \%$ el consumo de atenolol, y con lo cual invierte el perfil institucional del consumo. Alternativamente, el Area Flores-Belén mostró con una proporción equivalente de consumo entre enalapril y atenolol. Asimismo, la variabilidad en la selección individual de los antihipertensivos conlleva a destacar la muy notable prescripción preferencial del atenolol en Palmares (45\%); en tanto en San Rafael de Heredia se empató la prescripción de la hidroclorotiacida con atenolol, y ambos superaron al enalapril por $+15 \%$.

El consumo mensual absoluto, de los antihipertensivos, reportado desde los diferentes sitios mostró diferencias en el curso del periodo analizado (Figura 2). De Palmares y Tibás se obtuvieron los perfiles mensuales más estables para los fármacos más prescritos; en tanto que la Clínica Solón Núñez y de San Rafael de Heredia mostraron un crecimiento evidente al comenzar el año, seguido de un consumo estable. Alternativamente, Flores-Belén mostró el perfil mensual más variable, sobretodo para atenolol y enalapril; mientras que, con estos mismos dos fármacos, en la clínica Carlos Durán se incrementó el consumo en el curso del tiempo.

\section{Discusión}

El presente trabajo se realizó con el propósito de revisar el empleo de los fármacos antihipertensivos disponibles en la SS y describir el tratamiento ambulatorio de la HTA a partir de los consumos los medicamentos al nivel institucional, así como comparar los perfiles de utilización de tales medicamentos entre clínicas con atención ambulatoria durante un periodo anual.

Los resultados de este estudio muestran que todos los fármacos antihipertensivos, disponibles en la SS para uso por vía oral, son empleados para tratar los pacientes atendidos en la consulta médica. Asimismo, con este estudio se demuestra que la utilización de cada medicamento, visto a partir del consumo individual absoluto y normalizado por medio de la DDD, es cuantitativamente diferente y varía de forma notable según la zona.

En términos de intervención farmacológica, todos los medicamentos categorizados como antihipertensivos disminuyen la presión arterial. No obstante, al amparo de los efectos a largo plazo, pocos medicamentos han probado su utilidad en términos de la salud pública; y en muchos casos, una combinación de drogas antihipertensivas es requerida para alcanzar el control. ${ }^{8,9}$ Entonces, es procedente la selección individual de la medicación más apropiada; ciertamente es preferible como monoterapia, aunque la combinación de fármacos puede ser requerida según la severidad de la enfermedad, de los resultados de los ensayos clínicos y de la presencia de una o varias condiciones mórbidas coexistentes. ${ }^{3,10}$

Entre los medicamentos disponibles para el tratamiento de la HTA, está documentado que los diuréticos y beta-bloqueadores disminuyen la mortalidad en pacientes con HTA.,11 En consecuencia, es recomendable iniciar la terapia con bajas dosis de diuréticos tiacídicos, bloqueadores beta-adrenérgicos o inhibidores de la enzima convertidora de la angiotensina I en II (ECA) como primera línea de tratamiento, mientras no exista contraindicación o no haya indicación expresa de otra alternativa; de modo que los antagonistas de calcio podrían reservarse para los pacientes que no responden o no toleran los anteriores. Sin embargo, es necesario individualizar la terapia según las características de los pacientes. Se recomiendan los inhibidores de la ECA para pacientes diabéticos (particularmente si cursa con albuminuria) o con insuficiencia cardiaca congestiva, disfunción ventricular izquierda, hipertrofia ventricular izquierda o fenómeno de Raynaud; los beta-bloqueadores para pacientes con migraña, glaucoma, hipertiroidismo o tremor esencial; y especialmente los "cardioselectivos" para aquellos que han tenido infarto miocárdico, o tienen angor, prolapso de válvula mitral o taquicardia ventricular. $3,10,11$ En caso de dislipidemias, los inhibidores de la ECA, los bloqueadores alfa, los antagonistas de canales de calcio y del receptor de angiotensina constituyen una buena elección pues no muestran efectos deletéreos sobre el perfil lipídico. ${ }^{10,11}$ En embarazadas, la hidralacina y la metildopa son los fármacos de elección; y para pacientes con osteoporosis o con urolitiasis, las drogas de elección son los diuréticos 


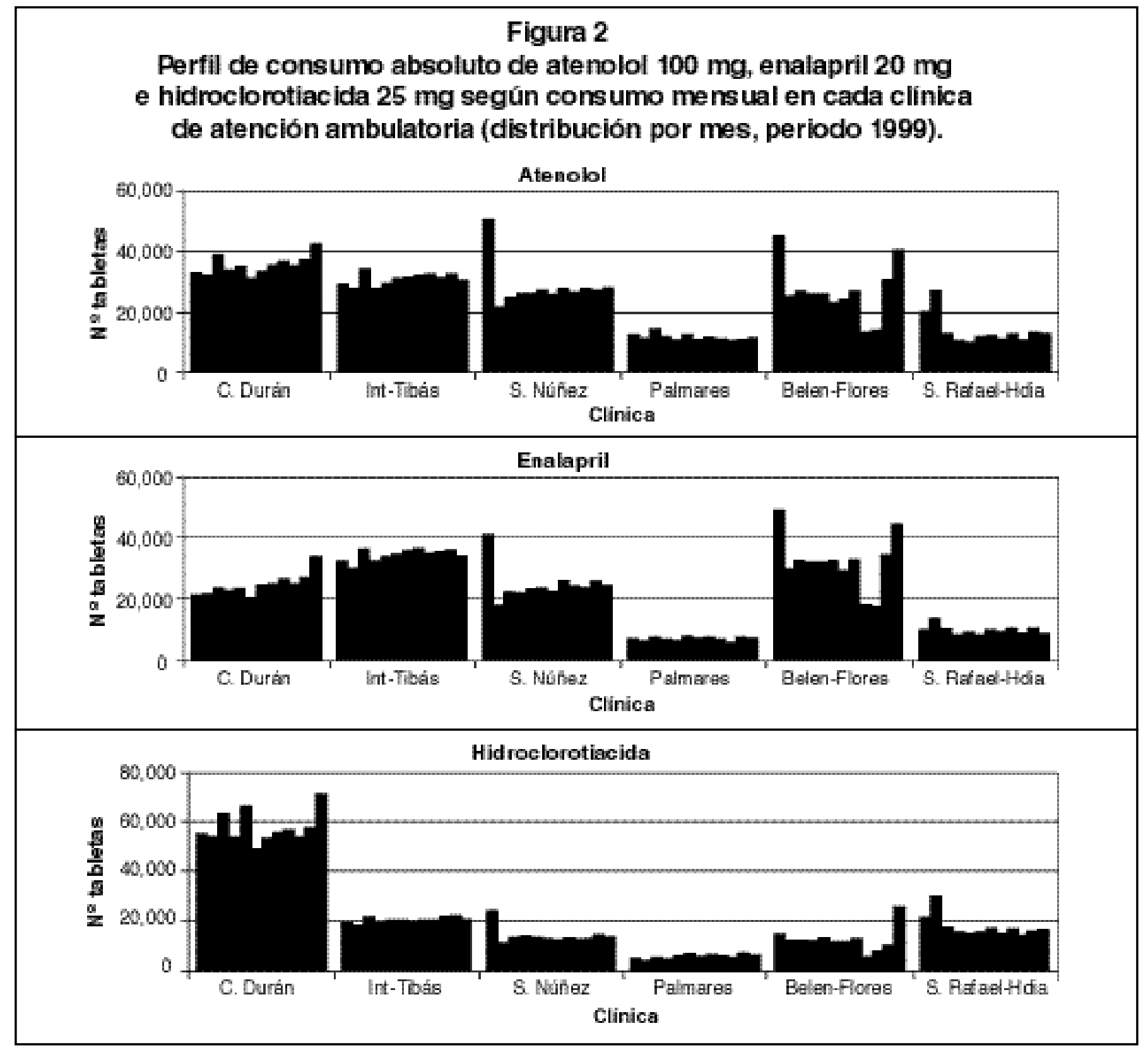

de tipo tiacídico. . $^{3,10,12}$ Además, la individualización de la terapia farmacológica se aplica a grupos poblacionales, dado que debe tenerse en cuenta que para pacientes de raza negra, los diuréticos y los antagonistas de canales de calcio son los más activos, ${ }^{11}$ en tanto que para pacientes mayores con HT sistólica aislada, se prefiere un diurético (tipo tiacida) con o sin el beta-bloqueador (pero no beta-bloqueador solo), o los antagonistas de canales de calcio con larga duración (tipo amlodipina). ${ }^{8-10,13-15}$ No cabe duda de que, en procura de maximizar el beneficio al paciente hipertenso según lo expuesto con anterioridad, disponer de una oferta variada de fármacos antihipertensivos es esencial.

Los resultados de este estudio demuestran un perfil diferencial de consumo entre los distintos antihipertensivos; evi- dencian la prescripción preferencial de los diuréticos y los beta-bloqueadores como primera elección, complementada con los inhibidores de la ECA (enalapril). Asimismo, los hallazgos muestran que los prescriptores que trabajan con la SS están al corriente de las recomendaciones internacionales para el manejo ambulatorio de la HTA, y que el uso que se les da a estos fármacos va acorde con los lineamientos propuestos para su uso racional.

En cuanto a los hábitos de prescripción global, la distribución del consumo actual de los antihipertensivos muestra un perfil diferencial respecto de los reportados en estudios previos; $y$ dos aspectos merecen ser destacados, la disminución del uso de la metil-dopa (y del propranolol), y el incremento de la utilización del enalapril (y de la hidroclorotiacida); esto últi- 
mo quizá debido a la más reciente introducción al mercado farmacéutico de los inhibidores de la ECA.

En cuanto a la metil-dopa, ahora muestra un consumo absoluto marginal pero un estudio previo sobre el manejo ambulatorio de la HTA documentó la prescripción preferencial de metildopa (27.6\%), seguida por el propranolol $25.0 \%$, atenolol $20.8 \%$ e hidroclorotiacida $12 \%{ }^{16}$ A pesar de que el estudio actual no separa a los beneficiarios por grupo etario, cabe mencionar que hace una década los pacientes mayores de 60 años, como destinatarios del $37 \%$ de toda la medicación prescrita, tenían a la metil-dopa con el $3^{\circ}$ lugar de mayor frecuencia de prescripción (precedida por ibuprofeno y antiácido de hidróxido de aluminio con magnesio), mientras que hidroclorotiacida ocupaba apenas un discreto sexto lugar, ${ }^{17} \mathrm{y}$ los beta-bloqueadores ni se citaron.

Por otra parte, el estudio anterior también documentaba que un $51 \%$ de los pacientes recibían monoterapia, con predominancia del uso de beta-bloqueadores y metil-dopa; y que un 46\% tenían tratamiento biasociado: hidroclorotiacida+metildopa $(45 \%)$ e hidroclorotiacida+beta-bloqueadores $(37 \%){ }^{16}$ Evidentemente, un abordaje farmacológico inicial como monoteraria es deseable, pero la posibilidad de combinar dos clases de antihipertensivos es una opción terapéutica real en el contexto de la SS.

Desafortunadamente, una debilidad de este tipo de estudios es la incapacidad para el registro de posibles combinaciones farmacológicas, lo cual constituye una limitante real cuando se aplica la DDD para analizar el consumo de los medicamentos. Ciertamente, la insensibilidad al uso combinado de medicamentos puede contribuir a una sobreestimación del número de pacientes hipertensos tratados a nivel institucional. Es decir, a partir del consumo institucional se ha estimado 185,639 pacientes si todos recibieran monoterapia.

Sin embargo, si se extrapolaran los datos actuales, por ejemplo, con el estudio que documentó sólo un $51 \%$ de los pacientes con monoterapía, entonces se tendrían unos 94,676 pacientes con monoterapia y unos 45,482 pacientes con terapia biasociada y una pequeña fracción con tratamiento triasociado. En consecuencia se podría sospechar que unos 140,158 pacientes estarían siendo beneficiados; pero esa cantidad representaría apenas un $4 \%$ de la población cubierta por la SS, y a menos de la mitad $(40 \%)$ de los hipertensos calculados a partir de una estimación conservadora de un $10 \%$ como prevalencia para HTA. En todo caso, los hallazgos sugieren que, en el mejor de los casos, una fracción importante de pacientes hipertensos podría ser tratada al margen de la SS (Medicina privada); ó bien, una gran proporción de enfermos podrían no estar controlada ni tratada del todo, con el agravante de que esa fracción podría ser aún más amplia, al amparo de las estimaciones extranjeras que señalan la prevalencia entre el 15 y $25 \%$ de la población. ${ }^{18,19}$

En este sentido, ya desde 1990 se había citado que la prevalencia general de la HTA era del 10 al 20\% de la población, específicamente, se planteó que sería de un $10 \%$ en la población costarricense; y entonces se estimaba que la cobertura del tratamiento antihipertensivo alcanzaba un $30 \%$ de los pacientes hipertensos del país. ${ }^{20}$ De acuerdo con las estimaciones actuales, se habría incrementado la cobertura global en $+10 \%$, pero aún es mejorable y, dado que las coberturas locales son variables y ninguna de las analizadas alcanza el 10\% de su población, es en este ámbito donde habría que reforzar los esfuerzos para ampliar el número de beneficiarios.

En conclusión los resultados de este estudio muestran que todos los fármacos antihipertensivos disponibles en la SS son requeridos por los prescriptores para el manejo de la HTA, y su utilización describe un perfil heterogéneo en cuanto al consumo general de cada compuesto. Los diuréticos, los beta-bloqueadores y los inhibidores de la ECA representan los fármacos con mayor prescripción institucional y local, lo cual está en concordancia con los lineamientos consensuales para el manejo de la HTA; la prescripción predominante de hidroclorotiacida y atenolol sugiere unos hábitos de prescripción acordes con el uso racional de los medicamentos. Los perfiles derivados de los centros de atención médica ambulatoria coinciden con la heterogeneidad institucional, aunque las variaciones en las preferencias de prescripción por medicamentos particulares son notables, especialmente en torno al enalapril. El consumo marginal de ciertos fármacos, como metildopa e hidralacina, demuestra que se reservan estos medicamentos como de segunda línea para una intervención farmacológica en casos especiales. Además, contrario a lo que se esperaba, debido a que la HTA es una patología crónica, el consumo individual de los antihipertensivos más usados en las distintas clínicas mostró variaciones notables en su curso temporal en algunos momentos, lo cual debe ser investigado para evaluar posibles factores influyentes. Finalmente, los datos sugieren que si bien muchas personas reciben tratamiento antihipertensivo con la $\mathrm{SS}$, en el país hay una fracción importante de personas hipertensas sin control y tratamiento, de modo que es pertinente dirigir esfuerzos para captar esos pacientes y ofrecer los beneficios institucionales a este grupo de población.

\section{Abstract}

Justification: Systemic hypertension is a chronic and asymptomatic disease. It is the first cause of ambulatory medical visits. It contributes directly to the first cause of mortality in the country, and it is almost always treatable with an integral intervention which includes pharmacological therapy.

Objective: To identify the most prescribed drugs at institutional level, to evaluate if the resultant profile is compatible with the principles of the rational use of antihypertensive drugs and to compare the use of these drugs in similar ambulatory medical care clinics during a year.

Methods: An pharmacoepidemilogic observational study type; a revision of institutional consumption was obtained of each antihipertensive drug during one year (1999), and local 
report of monthly consumption during 1999 of six ambulatory medical care clinics. The defined diary dose (DDD) was calculated and estimation of treated patients.

Results: Most prescribed drugs were atenolol (25\%), enalapril $(21 \%)$ and hidroclorotiazida (19\%). According to DDD, the global consumption of atenolol was $39 \%$, enalapril $25 \%$ and hidroclorotiacida $23 \%$, which benefits $87 \%$ of the patients treated. The antihypertensive drug treatment was given to about 185,639 patients. At local level, the consumption profiles tend to simulate the institutional distribution because of the predominant use of atenolol, but the diuretics are alternated with enalapril. We obtained variations in the mensual consumption during the period.

Conclusions: All antihipertensives drugs available are used although their consumption showed an heterogeneous profile. The drugs that were most prescribed at local level reflect in the national institutional profile, but there are remarkable local variations. The prescription of these drugs showed a rational use of antihypertensive drugs in the ambulatory management. The estimations derived from the consumption suggest that a great proportion of patients have no control, or are treated by private physicians.

\section{Agradecimientos}

A la Dra. Isabel Chaves de Palmares, Dra. Nora Benavides de Tibás, Dra. Laura Monge de la S. Núñez, Dra. Sara Vargas de C. Durán, Dra. Ligia Aguilar de Belén-Flores y Dr. Luis Diego Porras de San Rafael de Heredia, por su colaboración al brindar los datos del consumo local.

\section{Referencias}

1. Williams GH. Vasculopatía hipertensiva. En Isselbacher KJ, Braunwald E, Wilson JD, Martin JB, Fauci AS, Kasper DL (Eds.): Harrison Principios de Medicina Interna, tomo I. Madrid: McGraw-Hill Interamericana 1994: 1286 - 1303.

2. Sección de Información Biomédica. Cambios en la morbilidad y mortalidad por edad y sexo Costa Rica 1987, 1992 y 1997. San José: Caja Costarricense de Seguro Social, Serie Estadísticas de la Salud No. 8B, 1999: $101 \mathrm{pg}$.

3. The Sixth Report of the Joint National Committe on Prevention, Evaluation, and Treatment of High Blood Pressure. Arch Intern Med 1997, 157 (nov 24): 2413-2443.

4. Cada DJ, Covington TR, Hebel ST, Hussar DA, Lasagna L, Olin BR. Drug Facts and Comparisons, 1999 edition. St. Louis: Facts and Comparisons Inc.: 959-1060.

5. Comité Central de Farmacoterapia, Departamento de Farmacoterapia. Lista Oficial de Medicamentos. San José: Caja Costarricense de Seguro Social, 1999-2000: 60-61.

6. Informe de Consumo a Nivel Nacional, enero a diciembre de 1999. Sección de Contabilidad Bienes Muebles y Suministros, Dirección Financiero Contable. San José, Caja Costarricense de Seguro Social, 1999: $8 \mathrm{p}$

7. Anatomical Therapeutic Chemical (ATC) Classification Index and DDD. Oslo: WHO Collaboratig Centre for Drug Statistics Methodology 1996: 158 p.
8. World Health Organization (WHO). Guidelines for the treatment of hypertension. W.H.O. Drug Information 1999, 13(3): 157.

9. Ramsay LE, Williams, B, Johnston GD. Guidelines for management of hipertensión: report of the third working party of the British Hypertension Society. Journal of Human Hypertension 1999, 13: 569-592.

10. United States Pharmacopeial (USP DI) Hypertension. USP-DI Update volumes I and II, 1999 (may) : 1954 -1982.

11. Abramowics M. (Ed.) Drugs for hypertension. Drugs of choice from The Medical Letter. New York: The Medical Letter Inc., 1999: 93105 .

12. YeeYoung L1, Koda-Kimble MA, Gugliemo BJ, Kradjan WA. Essential Hypertension. In Handbook of Applied Therapeutics. Vancouver, Applied Therapeutics Inc., 1996: 8.1 - 8.14.

13. Messerli FH, Grossman E, Goldbourt U. Are b-blockers efficacious as first-line therapy for hypertension in the elderly?. JAMA 1998, 279(23): 1903-1907.

14. Beard K, Bulpitt C, Mascie-Taylor H. Management of elderly patients with sustained hypertension. Br Med J 1992, 304 (15 febr): 412-416.

15. MRC Working Part: Medical Research Council Trial of treatment of hypertension in older adults: principal results. Br Med J 1992, 304 (15 febr) : 405-12.

16. Sáenz-Campos D. Manejo terapéutico de la hipertensión arterial en pacientes ambulatorios. Fármacos 1990, 6(2): 53-59.

17. Sáenz-Campos D. Estudio comparativo de la prescripción de fármacos a pacientes mayores y menores de 60 años en la consulta externa de Medicina General. Fármacos 1990; 6 (1): 42-48.

18. Juillard J. Graded management of hypertension in adults. Rev Prescr 1995, 15 (150): 290-294.

19. Kaplan NM, Glifford RW. Choice of initial therapy for hipertension. JAMA 1996, 276 (2): 1577-1580.

20. Chaves A. Estudio de consumo de medicamentos en el sistema DDD en la Seguridad Social de Costa Rica en los años 1988-1989. Fármacos 1990, 6(2): 11-51. 